\title{
HMOS Sensing of Oxidizing Species in an Atmospheric Plasma Jet
}

\author{
Seán Kelly, ${ }^{*}$ Gurusharan Singh Gogna, \& Stephen Daniels \\ School of Electronic Engineering, Dublin City University, Ireland \\ *Address all correspondence to: Seán Kelly, E-mail: sean.kelly@dcu.ie
}

\begin{abstract}
A novel implementation of heated metal-oxide semi-conductor gas sensing (HMOS) is utilized to map the oxidizing species concentration in the effluent of a low-temperature plasma jet. Optical breadboard, posts and feet accessories are repurposed to position a small HMOS sensor accurately at coordinates in the plane of effluent emanating from the KinPEN MED ${ }^{\circledR}$ plasma jet, a commercial atmospheric plasma system. Operating with an argon carrier gas, oxidizing species concentrations of 240-11 ppb can extend $500 \mathrm{~mm}$ from the 1-mm-diameter nozzle encompassing a radius of $20 \mathrm{~mm}$. For jet operation with compressed air, larger oxidizing species concentrations up to $1100 \mathrm{ppb}$ were found surrounding the jet. This diagnostic approach shows potential for continuous and spatially resolved monitoring of collective concentrations of oxidizing/reducing reactive species $\left(\mathrm{O}_{3}, \mathrm{NO}_{x}, \mathrm{H}_{2} \mathrm{O}_{2}\right)$, which is of particular interest in characterizing treatment areas, informing dosage, and enabling control systems in emerging clinical and industrial application environments.
\end{abstract}

KEY WORDS: plasma medicine, plasma diagnostics, cold atmospheric pressure plasma, low temperature atmospheric pressure plasma jet

\section{INTRODUCTION}

Plasma-generated oxidizing species such as Ozone have been utilised for sterilization and antibacterial properties since the $19^{\text {th }}$ century. ${ }^{1}$ This utility has expanded recently to a wider family of plasma-generated species with promising bioactive capabilities. These species are often collectively referred to as reactive oxygen nitrogen species (RONS); they result from the higher enthalpy products of atmospheric gases (e.g., O, N, $\mathrm{O}_{3}, \mathrm{NO}$ ) precipitated via plasma generation. The field of "plasma biomedicine" has recently garnered considerable research interest, ${ }^{2-6}$ including potential for novel cancer therapies. ${ }^{7,8}$ Many prospective clinical treatments aim to manipulate the reduction-oxidation (REDOX) biochemistry of cells ${ }^{9}$ by administering RONS formed in a low-temperature plasma discharge at atmospheric pressure. Gas flows are typically employed to deliver RONS to nearby target surfaces. ${ }^{10-12}$

Implementation of optical absorption spectroscopy diagnostics to atmospheric plasma jets ${ }^{13}$ has generated a fundamental understanding of key reactive species; however, detection typically requires relatively "bulky" laser and spectrometry systems. Similarly, mass spectrometry systems ${ }^{14}$ at atmospheric pressures have a large footprint incorporating several differential pumping stages and a quadrupole arm. These systems are 
comparatively large in relation to the jet size $(\sim \mathrm{cm})$ and thus present significant practical challenges for deployment in application scenarios. The continuing miniaturization of heated metal-oxide semiconductor (HMOS) technology ${ }^{15-17}$ offers the potential for adaptable and low-cost diagnostics for future low-temperature plasma application scenarios. In situ HMOS sensors can potentially complement laboratory-based optical and particle diagnostics, enabling real-time monitoring and control in application environments.

HMOS technology along with electrochemical gas sensing is cross sensitive to oxidant/reductant species present at the sensor. The auditing of reactive species prior to sensor deployment using species selective laboratory-based methods (e.g., optical adsorption techniques, mass spectrometry) is therefore a critical precursor and has been successfully employed in numerous fundamental studies. HMOS technology allows combined oxidizing/reducing reactive species density to be measured and could provide a practical (in terms of cost and size) monitoring and/or control solution in many future application scenarios.

HMOS gas sensors exploit the relative change in resistance of a heated thin metaloxide layer $\left(\mathrm{SnO}_{2}, \mathrm{WO}_{3}, \mathrm{In}_{2} \mathrm{O}_{3}, \mathrm{ZNO}\right)$ in the presence of an oxidizing/reducing gas such as ozone. ${ }^{15,18,19}$ Adsorption on an n-type semi-conductor material such as $\mathrm{SnO}_{2}$ leads to a relative decrease in charge carriers (electrons) and a relative decrease in conductivity (increased resistance), ${ }^{19,20}$ which can be monitored via a DC circuitry. The range, reliability, and sensitivity of a particular thin-film oxide for gas sensing is dependent on the particular surface microstructure. ${ }^{20}$ The sophistication and reliability of HMOS gas sensors is an area of growing research interest driven in part by the need for long-term monitoring of air quality. This report aims to explore, in part, application of this technology to an atmospheric plasma jet system.

The NuWave MO35 sensor ${ }^{21}$ is a commercially available sensor based on HMOS technology that is employed here to map the effluent of a biomedical plasma jet. This sensor includes temperature-control circuitry to obtain accurate resistance measurements. The kINPen-MED ${ }^{22}$ is a commercially available plasma jet designed for use in medical applications such as wound healing and sterilization. ${ }^{23}$ This low-temperature corona source consists of a pin and plate geometry enclosed in a ceramic shower head for plasma generation. The pin electrode is biased at a $\mathrm{MHz}$ frequency $(1 \mathrm{MHz})$ with voltage amplitudes of the order of $2-6 \mathrm{kV}^{22,23}$ pulsed at a $\mathrm{kHz}$ frequency presumably to limit gas heating. ${ }^{24}$ In this report, the device is operated with compressed air and bottled argon ( $99.999 \%$ purity).

Optical absorption techniques in the ultraviolet and infrared bands have reported $\mathrm{O}_{3}$ and $\mathrm{NO}_{\mathrm{x}}$ species in close proximity to a plasma jet nozzle $(\sim 20 \mathrm{~mm}) \cdot{ }^{13,25-27}$ In this report, we explore the spatial variation in these oxidizing species concentration in the far regions $(25-500 \mathrm{~mm})$ for this jet. RONS species $\left(\mathrm{O}_{3}, \mathrm{NO}_{\mathrm{x}}, \mathrm{O}, \mathrm{N}\right)$ share a competitive chemistry that varies with position inside and outside the plasma region. ${ }^{26,28}$ Lowerenthalpy RONS $\left(\mathrm{O}_{3}, \mathrm{NO}_{\mathrm{x}}, \mathrm{H}_{2} \mathrm{O}_{2}\right)$ are generally longer lived and most likely to dominant outside the immediate region of the nozzle. 


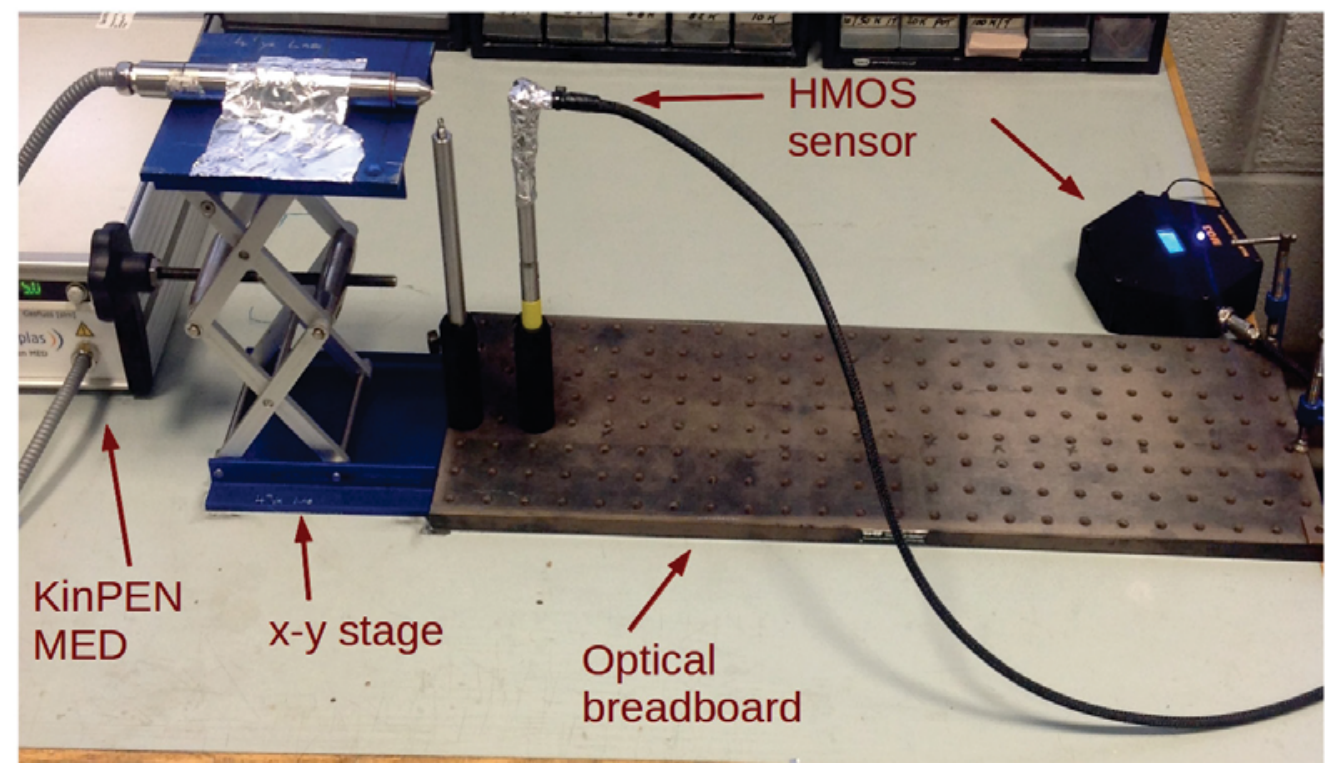

FIG. 1: KINPen-MED and NuWave MO3 sensor aligned with an optical breadboard.

\section{METHODS}

The plasma jet (kINPen-MED) and HMOS sensor (NuWave MO35) were laid out on a honeycomb structured optical breadboard as shown in Fig. 1. The kINPen-MED was aligned horizontally using an $\mathrm{x}-\mathrm{y}$ positioning stage. The sensor head (diameter $\sim 8 \mathrm{~mm}$ ) is aligned with the jet nozzle (diameter $\sim 1 \mathrm{~mm}$ ) at fixed positions on the breadboard using the optical post and feet accessories.

This novel setup allows spatially resolved measurement of long-lived reactive species in a plasma jet plume using a small-area HMOS sensor. An oxidizing species map is formed by positioning the sensor head at the numerous bore holes on the optical breadboard ( $25 \mathrm{~mm}$ separation). Finer readings are then taken by offsetting the plasma jet position relative to the breadboard bore holes within 5-20 $\mathrm{mm}$ and repeating the measurement. Data are output in parts per billion (ppb) increments each second with a maximum concentration detectable of $2000 \mathrm{ppb} .{ }^{21}$ Steady-state readings during jet operation were reached after $\approx 1$ minute. Sensor data are taken as an average over $\sim 60$ seconds of steady-state data capture. The error is calculated from the standard deviation of this data.

\section{RESULISAND DISCUSSION}

HMOS sensor measurements for operation of the kINPen-MED with an Argon carrier gas using a 5-slpm gas inlet flow are shown in Figs. 2 and 3. Background (ambient)

Volume 7, Issue 1, 2017 


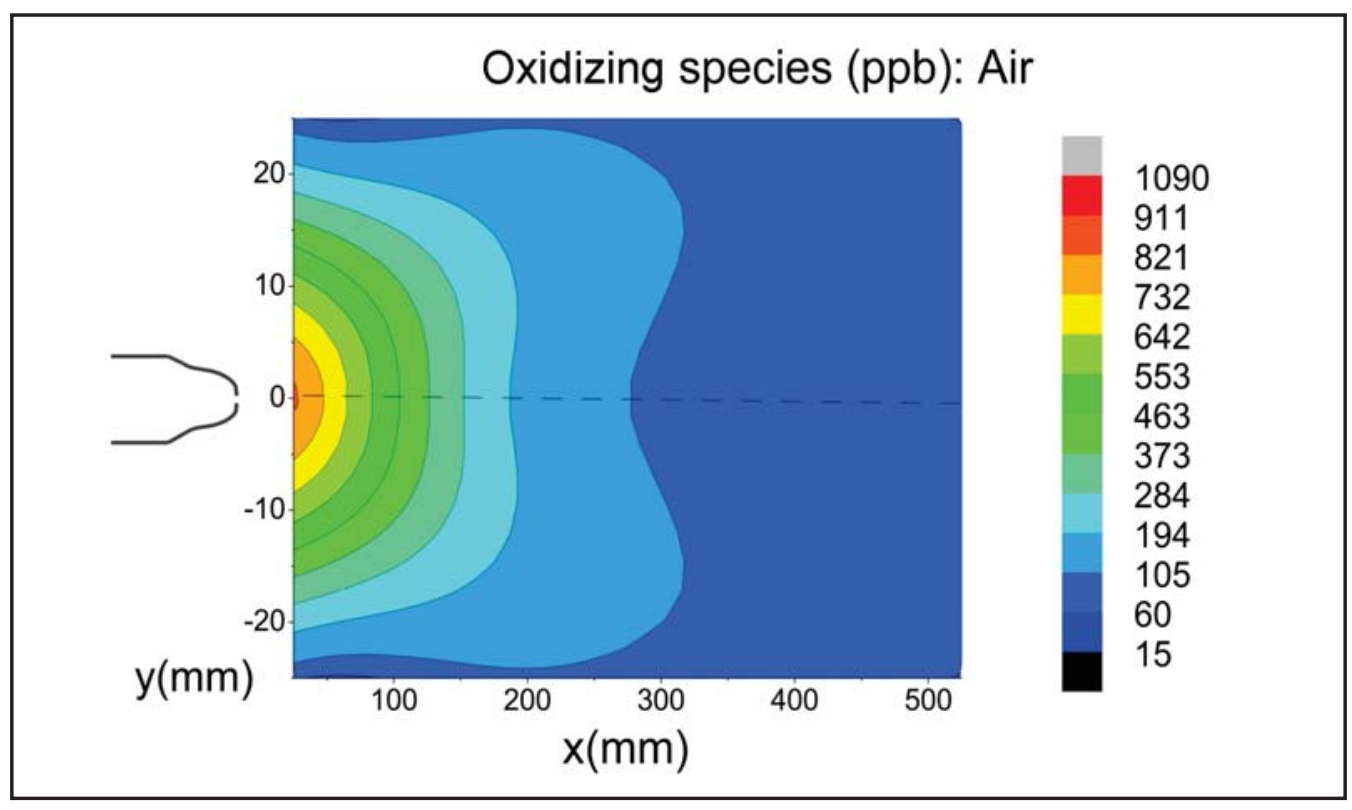

FIG. 2: Oxidizing species density (ppb) in the horizontal plane of plasma jet plume (25-500 $\mathrm{mm})$ : Argon (5 slpm inlet gas flow).

values of $\sim 11 \mathrm{ppb}$ were recorded. This value is typically constituent of ambient $\mathrm{O}_{3}$ and $\mathrm{NO}_{2}$ concentrations EPA16.

Figure 2 shows a relatively diffuse oxidizing species distribution around the plasma jet considering the nozzle diameter of $1 \mathrm{~mm}$. Peak oxidizing species values of $238 \mathrm{ppb}$ occur in the vicinity of the nozzle $(\mathrm{x}=25 \mathrm{~mm})$ and encompass a radius of $\sim 5 \mathrm{~mm}$. Downstream oxidizing species values of $\sim 30 \mathrm{ppb}$, well above the background density, occur at $\mathrm{x}=500 \mathrm{~mm}$ and encompass a radius of $\sim 25 \mathrm{~mm}$ surrounding the nozzle. Axial symmetry is clearly observable about the central axis here. Given the circular nozzle opening the axial symmetry shown in Figs. 2 and 3 can be expanded to characterize the oxidizing species distribution within the 3 -D plume (radial symmetry).

The spatial variation of oxidizing species concentration in Fig. 2 displays an (approximately) semicircular pattern with an expanding radius diffusing from the nozzle. Oxidizing species measurements along the central axis of the jet plume ( $\mathrm{y}=0$; see Fig. 2 ) are shown in Fig. 4. The oxidizing species concentration drops from $238 \pm 19.8 \mathrm{ppb}$ to $128 \pm 2.2 \mathrm{ppb}$ between $\mathrm{x}=25$ and $\mathrm{x}=100 \mathrm{~mm}$ away from the nozzle. At $200 \mathrm{~mm}$, oxidizing species values of $72 \pm 2.8 \mathrm{ppb}$ are recorded, and at $525 \mathrm{~mm}$ this drops to $27 \pm 2$ ppb. This trend displays an exponential rate of concentration decrease over this interval.

Figure 3 shows the HMOS sensor measurements for operation with air as the carrier gas $(5 \mathrm{slpm})$. Air is captured from the surrounding environment via a compressor (Clarke WizAir compressor) and fed to the KINPen-MED plasma jet system. Figure 3 shows a 


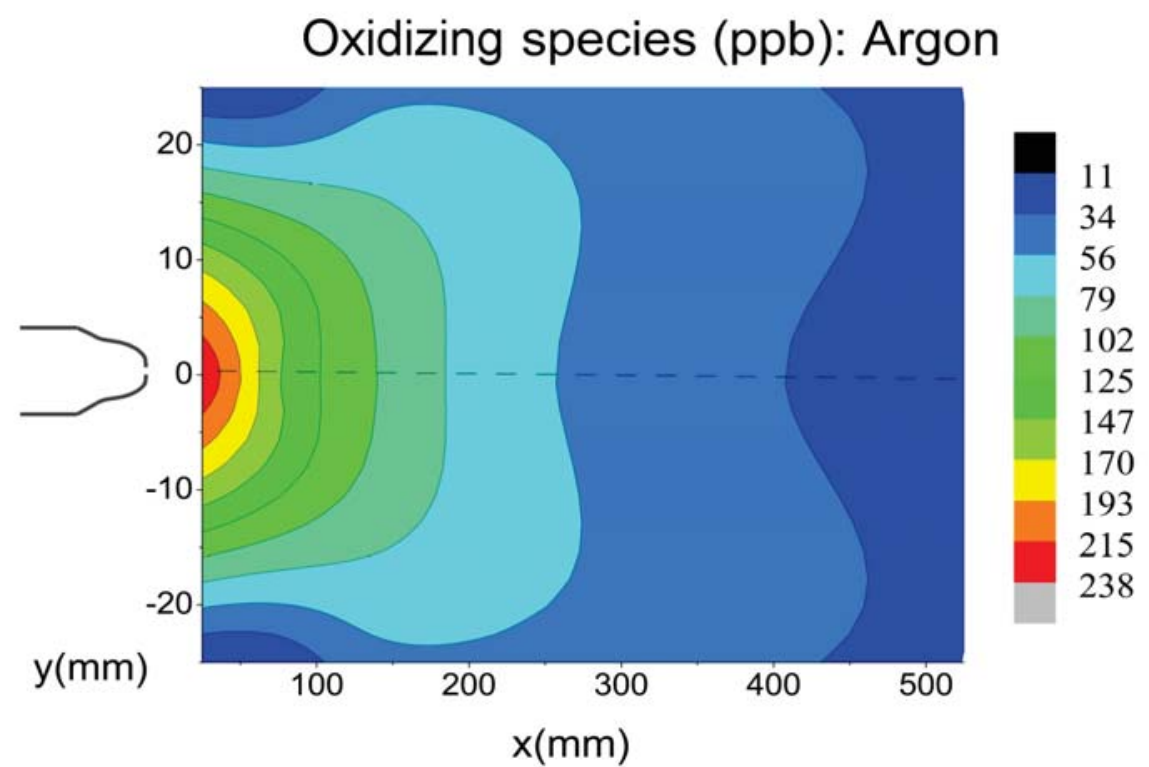

FIG. 3: Oxidizing species density (ppb) in the horizontal plane of plasma jet plume (25-500 $\mathrm{mm})$ : Air (5 slpm inlet).

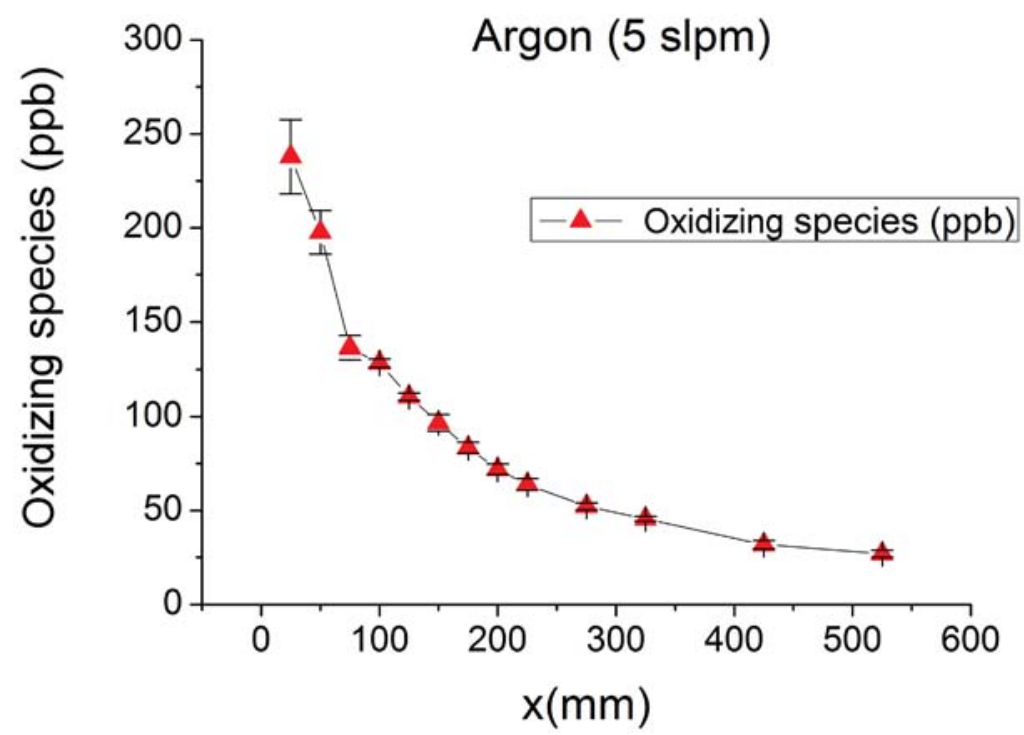

FIG. 4: Oxidizing species density (ppb) along the central axis ( $\mathrm{y}=0$ (see Fig. 2)): Argon (5 slpm inlet). 


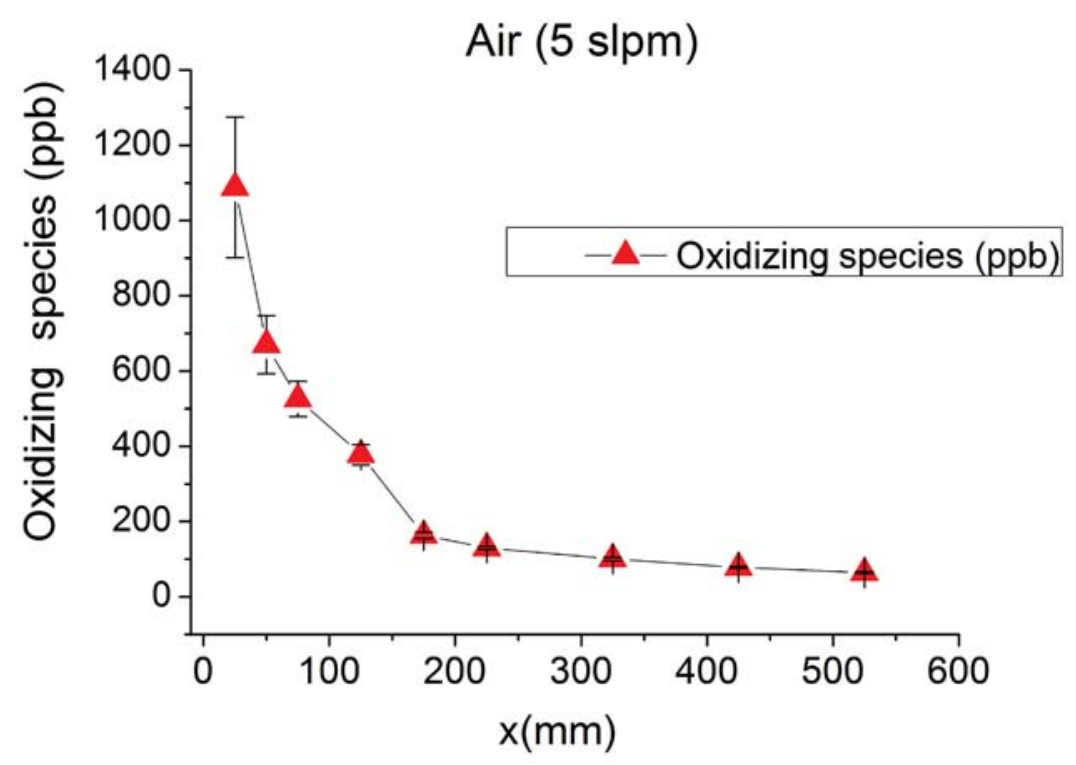

FIG. 5: Oxidizing species density (ppb) along the central axis (y = 0 (see Fig. 3)): Air (5 slpm inlet).

diffuse oxidizing species pattern similar to argon operation shown in Fig. 2; however, a significantly larger species concentration is measured here. In Fig. 5, oxidizing species density along the horizontal axis $(y=0)$ is given. At $\mathrm{x}=25 \mathrm{~mm}$, the oxidizing species concentration is $1088 \pm 187 \mathrm{ppb}$ which is approximately 5 times that of the equivalent argon value (Fig. 4). A similar exponential decay with increasing distance from the nozzle to argon operation is found for air. Oxidizing species values at $\mathrm{x}=125 \mathrm{~mm}, 225$ $\mathrm{mm}$, and $525 \mathrm{~mm}$ were $378 \pm 26.7,129 \pm 3.8$, and $64 \pm 2.4$, respectively (Fig. 3).

\section{CONCLUSION}

An exploratory application of heated metal-oxide sensor (HMOS) technology for reactive species detection in an atmospheric pressure plasma jet is reported. Spatial mapping of the oxidizing species concentration in the plume of the kINPen-MED plasma jet was carried out. A relatively diffuse oxidizing species distribution around the plasma plume for both argon and air carrier gases is found. Oxidizing species concentration decays exponentially along the central axis of the plasma plume, with concentrations exceeding background levels at over $500 \mathrm{~mm}$ downstream and within a $25-\mathrm{mm}$ lateral radius from the device nozzle. The peak oxidizing species density recorded for argon operation ranged from 27 to $238 \mathrm{ppb}$ along the central axis of the plume. Two-dimensional oxidizing species values of 240-211 ppb extend $500 \mathrm{~mm}$ from the nozzle, encompassing a radius of $20 \mathrm{~mm}$. Operation with compressed air led to significantly larger oxidizing species values ranging from 64 to $1088 \mathrm{ppb}$ along the central axis. Two-dimensional 
oxidizing species values of 1100-1115 ppb extended $500 \mathrm{~mm}$ from the nozzle within a radius of $20 \mathrm{~mm}$. Axial symmetry in the oxidizing species distribution occurred under both operating conditions. Given the circular nozzle opening design of the jet, radial symmetry can be asserted, and measurements can be extended to characterise the oxidizing species distribution within the 3-D plume.

\section{ACKNOWEDGMENIS}

The author would like to thank Dr. Kevin McDonnell and Dr. Denis Dowling of the University College Dublin for use of the KinPEN-MED plasma device and for helpful discussions on the topic. The author wishes to thank Dr. Ruairi Monahan of NuWave Sensors, Ltd., for helpful insights and discussion related to this report. The author wishes to thank Dr. Gurusharan Singh Gogna and Dr. Stephen Daniels for use of the NuWave probe and helpful discussions on the topic.

\section{REFERENCES}

1. Siemens W. Ueber die elektrostatische Induction und die Verz"ogerung des Stroms in Flaschendr"ahten. Annalen der Physik. 1857;178(9):66-122. Available from: http://dx.doi.org/10.1002/andp.185717 80905.

2. Graves DB. Low temperature plasma biomedicine: a tutorial review. Phys Plasmas. 2014;21(8):080901. Available from: http://dx.doi. org/10.1063/1.4892534.

3. von Woedtke T, Reuter S, Masur K, Weltmann KD. Plasmas for medicine. Phys Reps. 2013;530(4):291320. Available from: http://www.sciencedirect.com/science/article/pii/S0370157313001634.

4. Weltmann KD, Polak M, Masur K, von Woedtke T, Winter J, Reuter S. Plasma Processes and Plasma Sources in Medicine. Contrib Plasma Phys. 2012;52(7):644-54. Available from: http://dx.doi. org/10.1002/ctpp.201210061.

5. Fridman G, Friedman G, Gutsol A, Shekhter AB, Vasilets VN, Fridman A. Applied Plasma Medicine. Plasma Process Polymers. 2008;5(6):503-33. Available from: http://dx.doi.org/10.1002/ppap. 200700154.

6. O'Connor N, Cahill O, Daniels S, Galvin S, Humphreys H. Cold atmospheric pressure plasma and decontamination. Can it contribute to preventing hospital-acquired infections? J Hosp Infect. 2014;88(2):59-65.

7. Keidar M. Plasma for cancer treatment. Plasma Sources Sci Tech. 2015;24(3):033001. Available from: http://stacks.iop.org/0963-0252/24/i=3/a=033001.

8. Schlegel J, Köritzer J, Boxhammer V. Plasma in cancer treatment. Clin Plasma Medi. 2013;1(2):2-7. Available from: http://www. sciencedirect.com/science/article/pii/S2212816613000206.

9. Graves DB. The emerging role of reactive oxygen and nitrogen species in redox biology and some implications for plasma applications to medicine and biology. J Phys D: Appl Phys. 2012;45(26):263001. Available from: http://stacks.iop.org/0022-3727/45/i=26/a=263001.

10. Kelly S, Turner MM. Generation of reactive species by an atmospheric pressure plasma jet. Plasma Sources Sci Tech. 62014;23(6):065013. Available from: http://stacks.iop.org/0963-0252/23/i=6/ $\mathrm{a}=065013$.

11. Kelly S, Turner MM. Atomic oxygen patterning from a biomedical needle-plasma source. J Appl Phys. 2013;114(12):123301. Available from: http://scitation.aip.org/content/aip/journal/jap/114/ $12 / 10.1063 / 1.4821241$.

12. Kelly S, Golda J, Turner MM, von der Gathen VS. Gas and heat dynamics of a micro-scaled atmo-

Volume 7, Issue 1, 2017 
spheric pressure plasma reference jet. J Phys D: Appl Phys. 2015;48(44):444002. Available from: http://stacks.iop.org/0022-3727/48/i=44/a=444002.

13. Reuter S, Sousa JS, Stancu GD, van Helden JPH. Review on VUV to MIR absorption spectroscopy of atmospheric pressure plasma jets. Plasma Sources Sci Tech. 2015;24(5):054001. Available from: http://stacks.iop.org/0963-0252/24/i=5/a=054001.

14. Grosse-Kreul S, Hubner S, Schneider S, Ellerweg D, von Keudell A, Matejčík S, Benedikt J. Mass spectrometry of atmospheric pressure plasmas. Plasma Sources Science and Technology. 2015;24(4):044008. Available from: http://stacks.iop.org/0963-0252/24/i=4/a=044008.

15. Das S, Jayaraman V. SnO2: A comprehensive review on structures and gas sensors. Prog Mater Sci. 2014;66:112-255. Available from: http://www.sciencedirect.com/science/article/pii/S0079642514000 565 .

16. Devan RS, Patil RA, Lin JH, Ma YR. One-dimensional metal-oxide nanostructures: recent developments in synthesis, characterization, and applications. Adv Funct Mater. 2012;22(16):3326-70. Available from: http://dx.doi.org/10.1002/adfm.201201008.

17. Comini E, Baratto C, Faglia G, Ferroni M, Vomiero A, Sberveglieri G. Quasi-one dimensional metal oxide semiconductors: preparation, characterization and application as chemical sensors. Prog Mater Sci. 2009;54(1):1-67. Available from: http://www.sciencedirect. com/science/article/pii/ S0079642508000534.

18. Liu X, Cheng S, Liu H, Hu S, Zhang D, Ning H. A survey on gas sensing technology. Sensors. 2012;12(7):9635. Available from: http://www.mdpi.com/1424-8220/12/7/9635.

19. Fine GF, Cavanagh LM, Afonja A, Binions R. Metal oxide semiconductor gas sensors in environmental monitoring. Sensors. 2010;10(6):5469. Available from: http://www.mdpi.com/1424-8220/10/6/5469.

20. Naisbitt SC, Pratt KFE, Williams DE, Parkin IP. A microstructural model of semiconducting gas sensor response: The effects of sintering temperature on the response of chromium titanate (CTO) to carbon monoxide. Sensors Actuators B: Chem. 2006;114(2):969-977. Available from: http://www.sciencedirect.com/science/article/pii/S0925400505007410.

21. Ozone sensor by NuWave sensors Ltd M. DCU Innovation Campus, Glasnevin, Dublin 11, Ireland. 2016; Available from: www.nuwaveventures.com.

22. kINPen-MED by Neoplas-tools GmbH. INP Greifswald e.V. 2016; Available from: http://www.neoplas-tools.eu/kinpen-med.html.

23. Weltmann KD, Kindel E, Woedtke TV, Hahnel M, Stieber M, Brandenburg R. Atmospheric-pressure plasma sources: prospective tools for plasma medicine. Pure Appl Chem. 2010;82(6). Available from: https://doi.org/10.1351/PAC-CON-09-10-35.

24. Kelly S, Turner MM. Power modulation in an atmospheric pressure plasma jet. Plasma Sources Sci Tech. 2014;23(6):065012. Available from: http://stacks.iop.org/0963-0252/23/i=6/a=065012.

25. Reuter S, Winter J, Iseni S, Peters S, Schmidt-Bleker A, Dünnbier M, Schäfer J, Foest R, Weltmann K-D. Detection of ozone in a MHz argon plasma bullet jet. Plasma Sources Sci Tech. 2012;21(3):034015. Available from: http://stacks.iop.org/0963-0252/21/i=3/a=034015.

26. Shimizu T, Sakiyama Y, Graves DB, Zimmermann JL, Morfill GE. The dynamics of ozone generation and mode transition in air surface microdischarge plasma at atmospheric pressure. New J Phys. 2012;14(10):103028. Available from: http://stacks.iop.org/1367-2630/14/i=10/a=103028.

27. Reuter S, Winter J, Iseni S, Schmidt-Bleker A, Dunnbier M, Masur K, Wende K, Weltmann K-D. The influence of feed gas humidity versus ambient humidity on atmospheric pressure plasma jet-effluent chemistry and Skin Cell viability. IEEE Trans Plasma Sci. 2015 Sept;43(9):3185-92. Available from: https://doi.org/10.1109/TPS.2014.2361921.

28. Gaens WV, Bogaerts A. Kinetic modelling for an atmospheric pressure argon plasma jet in humid air. J Phys D: Appl Phys. 2013;46(27):275201. Available from: http://stacks.iop.org/0022-3727/46/i=27/ $\mathrm{a}=275201$.

29. Environmental Protection Agency Aqdf. Dublin, Ireland. 2016; Available from: http:/www.epa.ie/air/ quality/. 